\title{
Clinical Characteristics of Patients with COVID-19 in Saudi Arabia - A Single Center Experience
}

\author{
Sarfraz Saleemi ${ }^{*}$, Mohammed Alhajji ${ }^{1}$, Reem Almaghrabi ${ }^{2}$, Fahad Alrabiah ${ }^{2}$, Faisal Albaiz ${ }^{1}$, Rayan \\ Alfadda ${ }^{3}$ and Jameela Edathod $u^{4}$
}

\section{${ }^{1}$ Section of Pulmonary Medicine, Department of Medicine, King Faisal Specialist Hospital \& Research Center, Saudi Arabia}

${ }^{2}$ Section of Infectious Diseases, Department of Medicine, King Faisal Specialist Hospital \& Research Center, Saudi Arabia ${ }^{3}$ Section of Rheumatology, Department of Medicine, King Faisal Specialist Hospital \& Research Center, Saudi Arabia

${ }^{4}$ Section of Internal Medicine, Department of Medicine, King Faisal Specialist Hospital \& Research Center, Saudi Arabia

\begin{abstract}
Background: Saudi Arabia announced its first case of novel coronavirus (COVID-19) on March 2, 2020. We report relevant clinical features and outcome of 51 patients admitted with laboratory confirmed COVID-19 infection.

Methods: This is a retrospective review of consecutive patients admitted with diagnosis of COVID-19 confirmed by Real Time Reverse Transcription Polymerase Chain Reaction (RT-PCR). Clinical, laboratory, radiology and treatment outcome data were obtained by reviewing electronic medical record.

Results: Median $(\mathrm{Q} 1, \mathrm{Q} 3)$ age was $49(30,66)$ years and twenty-eight $(55 \%)$ patients were female. The most common symptoms were cough (69\%), fatigue (67\%), fever (63\%), Anorexia (37\%), headache $(29 \%)$ and dyspnea (27\%). Thirty $(59 \%)$ patients had mild disease while $21(41 \%)$ were categorized as severe. The most notable laboratory findings were lymphopenia, high neutrophil to lymphocyte ratio and elevated levels of C-reactive protein, ferritin and D-dimer. Twenty-three (45\%) patients had normal chest imaging, 6 (12\%) had focal and 22 (43\%) bilateral opacities. Forty-four (86\%) patients received combination of azithromycin and hydroxychloroquine, 14 (27\%) antiviral Lopinavir/Ritonavir (Kaletra) and Ribavirin, either alone or in combination and 24 (47\%) broad spectrum antibiotics. Seven (14\%) patients were transferred to the intensive care unit. Three patients required invasive mechanical ventilation of which two had successful extubation. Two patients died of which one had "do not resuscitate order" and was not intubated. Thirty-five $(69 \%)$ patients recovered. Mean ( \pm SD) duration of time to negative COVID-19 RT-PCR from symptom onset was 21 ( \pm 7.3) days.
\end{abstract}

Conclusion: This is the first study of COVID-19 cohort in Saudi Arabia. Cough, fatigue, fever, anorexia, headache and dyspnea were the most common symptoms. Twenty-one (41\%) patients had severe disease. Thirty-five (69\%) patient recovered and there were 2 (4\%) deaths.

\section{Keywords}

COVID-19, SARS-CoV-2, Infection, Polymerase chain reaction, Chest imaging

\section{Introduction}

The severe acute respiratory syndrome coronavirus 2 (SARS CoV-2) causes coronavirus disease 2019 (COVID-19), which has become a global pandemic since the first cases were reported in December 2019 in Wuhan, China and as of April $30^{\text {th }}, 2020$ it has spread across the globe affecting more than 3 million people and $>200,000$ deaths worldwide $[1,2]$. COVID-19 is a betacorona virus in the same subgenus as the severe acute respiratory syndrome (SARS) and has been shown to use the same receptor, the angiotensin-converting enzyme 2 (ACE2), for cell entry [3,4]. SARS-CoV2 infection can lead to severe respiratory disease leading to Acute Respira-
*Corresponding author: Sarfraz Saleemi, Section of Pulmonary Medicine, Department of Medicine MBC 46, King Faisal Specialist Hospital \& Research Center, Riyadh 11211, PO Box 3354, Saudi Arabia, Tel: +966-503194569, Fax: +966-11-4427499

Received: May 23, 2020

Accepted: June 10, 2020

Published online: June 12, 2020

Citation: Saleemi S, Alhajji M, Almaghrabi R, et al. (2020) Clinical Characteristics of Patients with COVID-19 in Saudi Arabia - A Single Center Experience. Res Rev Infect Dis 3(1):68-74 
tory Distress Syndrome (ARDS) similar to SARS-CoV infection with high mortality [5]. The symptoms of COVID-19 vary from dry cough, fever and shortness of breath to severe respiratory insufficiency and ARDS. Around $81 \%$ are found to have a mild disease, $14 \%$ severe and $5 \%$ critical in a large series of confirmed cases of COVID-19 [6]. SARS-CoV-2 induced pneumonia and respiratory failure is associated with activation of various inflammatory cytokines in particular interleukin 6 (IL-6). People with advanced age and those with co-morbid conditions are at risk of developing severe complications and increased mortality [7]. Several laboratory parameters have poor prognostic significance including lymphopenia, elevated levels of C-reactive protein, ferritin, lactate dehydrogenase, D-dimer, creatinine kinase (CK) and liver enzymes [8,9]. Typical radiology features include ground glass opacities either focal or diffuse depending upon the severity of lower respiratory involvement [10]. There is currently no approved therapy for COVID-19. Several off-label therapeutic agents are being used although there is no sufficient evidence for their use. Infectious Disease Society of America recommend their use only as part of clinical trial [11]. This retrospective study, the first from Saudi Arabia, shed the light on clinical, laboratory and radiology characteristics and outcome of COVID-19 patients.

\section{Methods}

\section{Study population, setting, and data collection}

Consecutive adult patients $\geq 18$ year of age admitted to a tertiary care hospital from March 13, 2020 onward were included in the study. Definite case was defined as a patient with laboratory confirmation of SARS CoV-2 virus (COVID-19 infection) by real time reverse transcriptase polymerase chain reaction (RT-PCR) irrespective of clinical signs and symptoms. Patients $<18$ year of age, suspected cases with symptoms but without evidence of laboratory confirmation of SARS-CoV2 infection and patient with positive SARS-CoV2 PCR but discharged to home isolation without being admitted to hospital were not included in the study. The data comprising of demographics, epidemiological, clinical, laboratory and radiology characteristics as well as treatment outcome was abstracted from electronic medical record of the patients by expert research coordinators and cross checked by investigators. The data collection was formulated on REDCap (Research Electronic Data Capture) platform regulated by Research Advisory Council of the institution and confidentiality is insured by restricting access, which is password protected, to only approved projects.

\section{Clinical data}

Information on patient demographics, symptoms onset, travel and contact history, clinical symptoms, co-morbid medical conditions and severity of disease were documented. Severity of disease was classified into four groups as per institution guidelines. Stage A- patient with no clinical signs or symptoms but positive COVID-19 RT-PCR, stage B- patients with upper respiratory tract infection symptoms and/ or other mild symptoms including fever and gastrointestinal symptoms without evidence of pneumonia, stage C- patients with radiology evidence of lower respiratory tract infection or hypoxemia with oxygen saturation $\leq 93 \%$ at rest breathing room air or drop in oxygenation compared to baseline but not requiring intensive care unit (ICU) admission and stage D was categorized as patients requiring ICU admission due Respiratory rate of 30 breaths/min, arterial oxygen partial pressure to fractional inspiratory oxygen ratio $\left(\mathrm{PaO}_{2} / \mathrm{FiO}_{2}\right)<$ 300 , more than $50 \%$ lung involvement on chest imaging or critical respiratory failure requiring mechanical ventilation, septic shock or multi-organ dysfunction. In this study, Stage $A$ and $B$ are categorized as Mild and Stage $C$ and D as severe disease. Acute Respiratory Distress Syndrome (ARDS) was defined as acute hypoxemic respiratory failure with ratio of the partial pressure of arterial oxygen to fraction of inspired oxygen $\left(\mathrm{PaO}_{2}: \mathrm{FiO}_{2}\right)<300$ and bilateral opacities on chest imaging which is not explained by heart failure or fluid overload. Vital signs including temperature, heart rate, respiratory rate, systolic blood pressure and oxygen saturation by pulse oximetry were documented. Data about therapeutic intervention including antiviral, anti-inflammatory and antibiotics therapy and clinical outcome such as discharge, transfer to intensive care unit and assisted ventilation was tabulated. Cure was defined as two negative COVID-19 RT-PCR 24 hours apart along with resolution of symptoms and clinical syndrome.

\section{Laboratory and radiology assessment}

Diagnosis of COVID-19 was confirmed by Real Time Reverse Transcription Polymerase Chain Reaction (RT-PCR) using nasopharyngeal swab in the hospital laboratory. PCR testing kits by Altona (Hamburg-Germany) approved by government regulatory authorities were used. Throat swab was also sent for multiplex PCR for common respiratory viruses and atypical bacteria using QIAstat kits by Qiagen (Hilden-Germany) and sputum culture where indicated to exclude co-infection. Clinical laboratory investigations included complete blood count, differential blood count including absolute neutrophil to lymphocyte ratio, chemistry panel including renal and hepatic function, inflammatory markers such as C-reactive protein (CRP), ferritin, pro-calcitonin were tested. Other laboratory parameters included D-dimer, Lactate dehydrogenase (LD), coagulation profile and creatinine kinase. Radiology data including plain chest X-ray and computed tomography (CT) scan of chest was included in the assessment.

\section{Statistical analysis}

Data was summarized by using descriptive statistics. Results were reported as median with inter-quartile range and mean with standard deviation and categorical variable were calculated as counts ( $n$ ) and percentages (\%). Difference between groups was analyzed using Mann-Whitney $U$ test, T-test and two tailed Z-test where appropriate.

\section{Ethical consideration}

The study was granted approval by the institutional review board and written informed consent was waived in view of retrospective non-interventional study.

\section{Results}

\section{Demographic and characteristics of patients}


Citation: Saleemi S, Alhajji M, Almaghrabi R, et al. (2020) Clinical Characteristics of Patients with COVID-19 in Saudi Arabia - A Single Center Experience. Res Rev Infect Dis 3(1):68-74

Table 1: Baseline demographic and clinical characteristics.

\begin{tabular}{|c|c|c|c|c|}
\hline \multirow{2}{*}{ Overall } & & \multicolumn{3}{|c|}{ Disease severity, n (\%) } \\
\hline & & Mild & Severe & $P$ value \\
\hline \multicolumn{2}{|l|}{$n=51$} & $30(59)$ & $21(41)$ & \\
\hline \multicolumn{5}{|l|}{ Demographic } \\
\hline Age, years median (Q1, Q3) & $48(30,66)$ & $40(27,58)$ & $61(49,76)$ & 0.0067 \\
\hline$\leq 40$ years $n(\%)$ & $18(35)$ & $15(50)$ & $3(14)$ & 0.0085 \\
\hline $41-60$ years $n(\%)$ & $16(31)$ & $9(30)$ & $7(33)$ & 0.8025 \\
\hline$>60$ years $n(\%)$ & $17(33)$ & $6(20)$ & $11(52)$ & 0.0159 \\
\hline \multicolumn{5}{|l|}{ Gender, n (\%) } \\
\hline Female & $28(55)$ & $21(70)$ & $7(33)$ & 0.0096 \\
\hline Male & $23(45)$ & $9(30)$ & $14(67)$ & 0.0096 \\
\hline $\mathrm{BMI}^{*}, \mathrm{~kg} / \mathrm{m}^{2}$, median $(\mathrm{Q} 1, \mathrm{Q} 3)$ & $28(26,32)$ & $28(23,31)$ & $29(26,32)$ & 0.61 \\
\hline Smoking past or present, $\mathrm{n}(\%)$ & $11(22)$ & $4(14)$ & $7(32)$ & 0.1211 \\
\hline Health care worker, n (\%) & $3(6)$ & $2(7)$ & $1(6)$ & 0.7263 \\
\hline \multicolumn{5}{|l|}{ Comorbidities, $n$ (\%) } \\
\hline Hypertension & $21(41)$ & $6(21)$ & $15(68)$ & 0.0006 \\
\hline Diabetes & $9(18)$ & $1(3)$ & $8(36)$ & 0.0022 \\
\hline Cardio vascular disease & $9(18)$ & $0(0)$ & $9(41)$ & 0.0001 \\
\hline Chronic lung diseases (COPD, BA, ILD) & $8(16)$ & $6(21)$ & $2(9)$ & 0.2584 \\
\hline Malignancy & $1(2)$ & $0(0)$ & $1(6)$ & 0.246 \\
\hline Chronic kidney disease & $6(12)$ & $0(0)$ & $6(27)$ & 0.0027 \\
\hline CTD“/immunosuppressed & $4(8)$ & $1(3)$ & $3(14)$ & 0.1802 \\
\hline$\geq 2$ comorbidities, $\mathrm{n}(\%)$ & $20(39)$ & $6(21)$ & $14(64)$ & 0.0018 \\
\hline \multicolumn{5}{|l|}{ Symptomatology, n (\%) } \\
\hline Fever & $32(63)$ & $15(52)$ & $17(77)$ & 0.0614 \\
\hline Cough & $35(69)$ & $19(66)$ & $16(72)$ & 0.5823 \\
\hline Dyspnea & $14(27)$ & $6(21)$ & $8(36)$ & 0.2149 \\
\hline Hemoptysis & $1(2)$ & $0(0)$ & $1(6)$ & 0.246 \\
\hline Sore throat & $21(41)$ & $13(45)$ & $8(36)$ & 0.5418 \\
\hline Fatigue & $34(67)$ & $19(66)$ & $15(68)$ & 0.8418 \\
\hline Anorexia & $19(37)$ & $11(38)$ & $8(36)$ & 0.9124 \\
\hline Diarrhea & $6(12)$ & $3(10)$ & $3(14)$ & 0.7188 \\
\hline Nausea/vomiting & $2(4)$ & $0(0)$ & $2(9)$ & 0.0969 \\
\hline Anosmia & $8(16)$ & $6(21)$ & $2(9)$ & 0.2584 \\
\hline Ageusia & $11(22)$ & $8(28)$ & $3(14)$ & 0.2301 \\
\hline Headache & $15(29)$ & $8(28)$ & $7(32)$ & 0.7414 \\
\hline \multicolumn{5}{|l|}{ Vital signs } \\
\hline Temperature ${ }^{\circ} \mathrm{C}$, median $(\mathrm{Q} 1, \mathrm{Q} 3)$ & $37.4(36.6,38)$ & $37(36.8,37.6)$ & $37.8(37.4,38.7)$ & 0.00008 \\
\hline$<38(\%)$ & $38(75)$ & $26(90)$ & $12(55)$ & 0.0043 \\
\hline$\geq 38(\%)$ & $13(25)$ & $3(10)$ & $10(45)$ & 0.0043 \\
\hline MAP $*+*$, mmHg, median $(\mathrm{Q} 1, \mathrm{Q} 3)$ & $86(79,95)$ & $85(77,97)$ & $88(81,92)$ & 0.3173 \\
\hline Heart rate, per minute, median $(\mathrm{Q} 1, \mathrm{Q} 3)$ & $80(74,88)$ & $80(74,88)$ & $81(74,94)$ & 0.4839 \\
\hline Respiratory rate, per minute, median $(\mathrm{Q} 1, \mathrm{Q} 3)$ & $20(20,20)$ & $20(20,20)$ & $20(20,26)$ & 0.0734 \\
\hline Oxygen saturation, \% median (Q1,Q3) & $97(95,99)$ & $98(97,99)$ & $95(91,97)$ & 0.0003 \\
\hline
\end{tabular}

*BMI: Body Mass Index; ${ }^{* *}$ CTD: Connective Tissue Disease; ${ }^{* * *}$ MAP: Mean Arterial Blood Pressure.

Sum of decimals in percentage values have been rounded to whole number where appropriate. The sum of percentages may not be equal to 100 . 
Citation: Saleemi S, Alhajji M, Almaghrabi R, et al. (2020) Clinical Characteristics of Patients with COVID-19 in Saudi Arabia - A Single Center Experience. Res Rev Infect Dis 3(1):68-74

Fifty-one consecutive patients admitted with confirmed diagnosis of COVDI-19 from March 13, 2020 onward were identified. The median (Q1, Q3) age of patients was 48 (30, $66)$ years. Twenty-eight (55\%) patients were female while 23 (45\%) were male. Demographic and clinical characteristics are summarized in Table 1. Eighteen (35\%) patients were $\leq$ 40 years, $16(31 \%)$ patients $41-60$ years and $17(33 \%)$ were > 60 years. Eleven (22\%) patients were either current or past smoker. Most common co-morbid medical condition was hypertension (41\%). Twenty (39\%) patients had $\geq 2$ co-morbid conditions. Most common symptoms were cough (69\%), fever (63\%), and dyspnea (27\%). Significant proportion of patients had extra-pulmonary symptoms including fatigue $(67 \%)$, anorexia (37\%), headache $(29 \%)$ ageusia $(22 \%)$ and anosmia (16\%). Median (Q1, Q3) temperature was 37.4 (36.6, $38){ }^{\circ} \mathrm{C}$ while $13(25 \%)$ had temperature $\geq 38{ }^{\circ} \mathrm{C}$. Median (Q1, Q3) $\mathrm{O}_{2}$ saturation (\%) was $97(95,99)$. Fifty-nine percent of patients had mild disease while $41 \%$ were categorized as severe. Patients with severe disease were more likely to be male compared to mid group ( $p=0.0096)$ and older with median $(\mathrm{Q} 1, \mathrm{Q} 3)$ age of $61(49,76)$ years, $(p=0.0067)$. Two or more co-morbid medical conditions were more common in severe group compared to mild ( $p=0.0018$ ) (Figure 1).

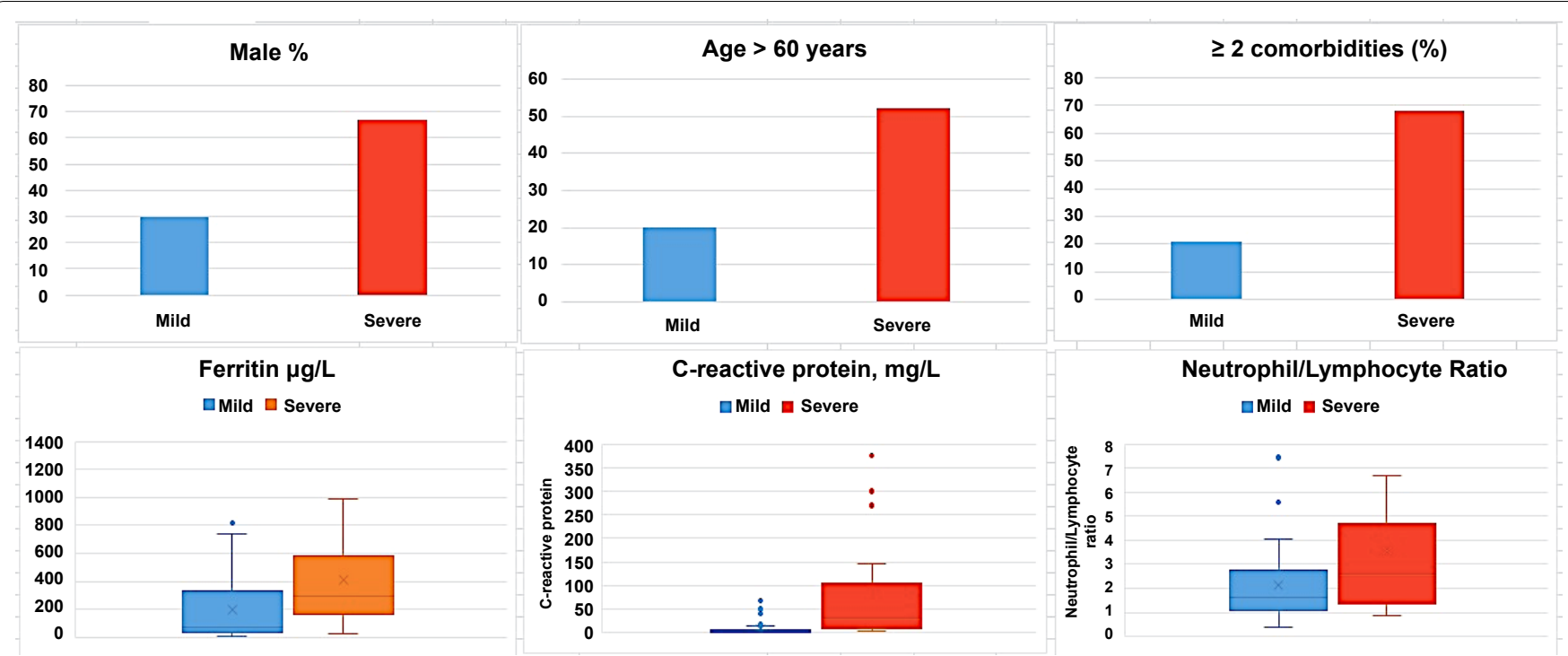

Figure 1: Risk factors for severe disease in COVID-19.

Severe disease compared to mild cases is associated with male gender, age $>60$ years, $\geq 2$ co-morbid medical conditions, higher ferritin, C-reactive protein and neutrophil/lymphocyte ratio.

Table 2: Baseline laboratory and radiologic features.

\begin{tabular}{|c|c|c|c|c|}
\hline & Overall & Mild & Severe & \\
\hline & $n=51$ & $\mathrm{n}=30$ & $\mathrm{n}=21$ & $P$ value \\
\hline Laboratory value, unit (normal value) & \multicolumn{4}{|l|}{ Median (Q1, Q3) } \\
\hline White blood cell, × 109/L, (3.9-11.0) & $5.31(3.72,6.12)$ & $5.23(3.68,5.90)$ & $5.31(4.14,7.88)$ & 0.1735 \\
\hline Neutrophil, $\times 10^{9} / \mathrm{L},(1.35-7.50)$ & $3.02(1.93,4.02)$ & $2.92(1.99,3.80)$ & $3.54(1.91,4.71)$ & 0.0637 \\
\hline Lymphocyte, $\times 10^{9} / \mathrm{L},(1.50-4.30)$ & $1.37(1.07,1.81)$ & $1.43(1.10,1.98)$ & $1.21(0.97,1.57)$ & 0.021 \\
\hline Neutrophil/Lymphocyte ratio, $(1.7 \pm 0.7)$ & $1.75(1.19,3.56)$ & $1.61(1.1,2.64)$ & $2.52(1.37,4.71)$ & 0.0285 \\
\hline Platelet count, $\times 10^{9} / \mathrm{L},(155-435)$ & $200(160,251)$ & $204(172,264)$ & $183(142,251)$ & 0.1498 \\
\hline Alanine aminotransferase, $U / L,(10-45)$ & $23(13,40)$ & $20(11,33)$ & $26(22,46)$ & 0.0989 \\
\hline Aspartate aminotransferase, U/L (10-45) & $25(18,38)$ & $21(17,30)$ & $34(21,50)$ & 0.0657 \\
\hline Albumin, g/L, (28-46) & $41(39,44)$ & $42(40,45)$ & $40(38,42)$ & 0.0784 \\
\hline Bilirubin, mmol/L, (0.0-21.0) & $6(3.8,9)$ & $5(3.3,7.6)$ & $7(4.8,10)$ & 0.0767 \\
\hline Creatinine, $\mu \mathrm{mol} / \mathrm{L},(46-96)$ & $67(57,86)$ & $63(56,76)$ & $79(63.2,107)$ & 0.0168 \\
\hline Creatine kinase, U/L, (24-195) & $85(56,129.5)$ & $69(47,97.5)$ & $107(63,177)$ & 0.0466 \\
\hline Lactate dehydrogenase, U/L, (135-214) & $220(184,276)$ & $208(178,235)$ & $266(209,422)$ & 0.0127 \\
\hline Prothrombin Time, (12.3-14.2) & $14.6(13.7,15.8)$ & $14.3(13.9,15.4)$ & $14.9(13.5,16)$ & 0.5028 \\
\hline D-dimer, $\mu \mathrm{g} / \mathrm{mL},(0.0-0.50)$ & $0.37(0.28,0.67)$ & $0.32(0.28,0.54)$ & $0.51(0.32,1.03)$ & 0.0455 \\
\hline
\end{tabular}


Citation: Saleemi S, Alhajji M, Almaghrabi R, et al. (2020) Clinical Characteristics of Patients with COVID-19 in Saudi Arabia - A Single Center Experience. Res Rev Infect Dis 3(1):68-74

\begin{tabular}{|c|c|c|c|c|}
\hline C-reactiveprotein, $\mathrm{mg} / \mathrm{L}, \quad(<3.0)$ & $5.9(2.05,30.9)$ & $2.6(0.9,6.45)$ & $28(9,106)$ & 0.0002 \\
\hline Ferritin, $\mu \mathrm{g} / \mathrm{L},(13.0-150)$ & $184(50,421)$ & $107(42,354)$ & $290(162,583)$ & 0.0089 \\
\hline \multicolumn{5}{|l|}{ Radiology features, $n$ (\%) } \\
\hline Normal & $23(45)$ & $23(77)$ & $0(0)$ & $<0.00001$ \\
\hline Focal & $6(12)$ & $5(14)$ & $1(5)$ & 0.1936 \\
\hline Bilateral & $22(43)$ & $2(7)$ & $20(95)$ & $<0.00001$ \\
\hline
\end{tabular}

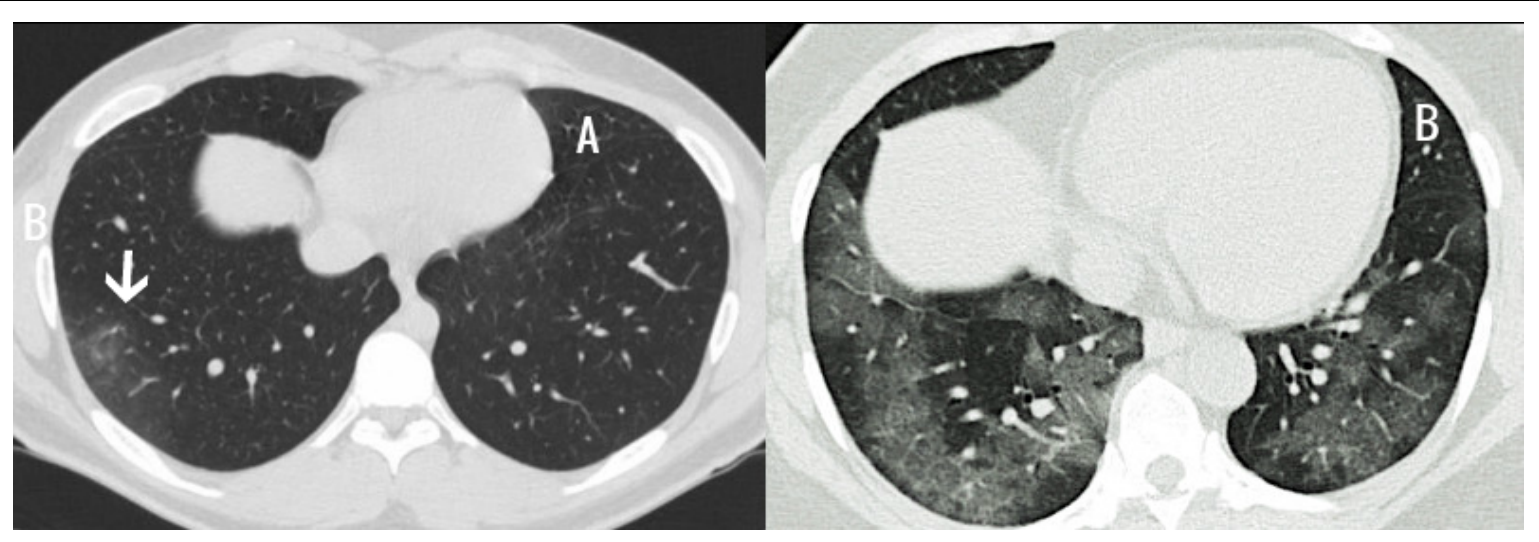

Figure 2: Focal and diffuse ground glass opacities in COVID-19 patients.

Thoracic CT scan of two patients with laboratory confirmed COVID-19. (A) Focal ground glass opacity (white arrow) in a 22-year-old male with mild symptoms and normal CRP and ferritin; (B) Diffuse bilateral ground glass opacities in a 48-year-old female with hypoxemia and elevated inflammatory markers.

\section{Laboratory and radiological features}

Important laboratory and radiological features along with differences in clinical severity groups are summarized in Table 2. The most common abnormal laboratory findings were lymphopenia, high neutrophil to lymphocyte ratio and elevated levels of C-reactive protein, ferritin and D-dimer. Patient with severe disease had more pronounced lymphopenia ( $p$ $=0.0210)$, higher levels of D-dimer $(p=0.0455)$, C-reactive protein (CRP) ( $p=0.0002)$, Lactate dehydrogenase (LD) ( $p=$ $0.0127)$ and creatine kinase (CK) $(p=0.0466)$. Multiplex PCR and respiratory culture revealed evidence of co-infection in only one patient whose sputum culture was positive for Moraxella Catarrhalis. All admitted patients had portable chest X-ray and 29 (57\%) had CT scan of chest. Most common abnormality on chest imaging was bilateral ground glass opacities 22 (43\%) (Figure 2). Twenty-three (45\%) patients had normal chest imaging.

\section{Therapeutic intervention}

Table 3 summarized the therapeutic management and clinical outcome of patients. Patients were managed according to the institutional COVID-19 guidelines which had updates during the study period due to the evolving evidence. The patients admitted in the early period received different treatment regimen than those admitted in the later study period. Forty-four (86\%) patients received combination of azithromycin and hydroxychloroquine while two patients had hydroxychloroquine alone. Twenty-four (47\%) patients were prescribed broad spectrum antibiotics. Fourteen (27\%) patients received antiviral treatment Lopinavir/Ritonavir (Kaletra) and Ribavirin, either alone or in combination. Five
Table 3: Treatment and clinical outcome.

\begin{tabular}{|l|l|}
\hline Total patients $\mathbf{n}=\mathbf{5 1}$ & $\mathbf{n}(\%)$ \\
\hline Treatment & \\
\hline Antiviral therapy & $19(37)$ \\
\hline Lopinavir/Ritonavir (Kaletra) & $12(63)$ \\
\hline Ribavirin & $7(37)$ \\
\hline Hydroxychloroquine & $46(90)$ \\
\hline Azithromycin & $44(86)$ \\
\hline Broad spectrum antibiotics & $24(47)$ \\
\hline Tocilizumab (IL-6* receptor blocker) & $5(10)$ \\
\hline Intravenous immunoglobulin therapy & $1(2)$ \\
\hline Intensive care unit (ICU) transfer & $7(14)$ \\
\hline Oxygen therapy & $4(57)$ \\
\hline Invasive ventilation & $3(43)$ \\
\hline ECMO & $0(0)$ \\
\hline Clinical outcome & \\
\hline Cured & $35(69 \%)$ \\
\hline Death & $2(4 \%)$ \\
\hline inpatient & $14(27 \%)$ \\
\hline ICU & $0(0)$ \\
\hline
\end{tabular}

Sum of decimals have been rounded to whole number where appropriate

*Interleukin-6, " Extracorporeal membrane oxygenation.

patients were treated with IL-6 receptor blocker, Tocilizum$\mathrm{ab}$ and one patient was given Intravenous Immunoglobulin (IVIG). 


\section{Comparison of disease severity}

Fifty-nine percent of patients had mild disease while $41 \%$ were categorized as severe. Patient with severe disease had more pronounced lymphopenia $(p=0.0210)$, higher levels of D-dimer $(p=0.0455), C$-reactive protein (CRP) $(p=0.0002)$, Lactate dehydrogenase (LD) $(p=0.0127)$ and creatine kinase (CK) $(p=0.0466)$ compared to mild group. Twenty patients $(95 \%)$ in severe group had bilateral opacities on chest imaging compared to $2(7 \%)$ in mild group ( $p=<0.00001)$.

\section{Clinical outcome}

Seven (14\%) patients were transferred to the intensive care unit. Three patients required invasive mechanical ventilation, two of whom had successful extubation. Two patients died of which one had "do not resuscitate order" and was not intubated. Thirty-five (69\%) patients recovered and were declared cured. Sixteen (31\%) patients were still at the hospital and none of them was in ICU at the time of conclusion of the study. Mean $( \pm S D)$ duration of time to negative COVID-19 RTPCR from symptom onset was 21 ( \pm 7.3 ) days (Table 3 ).

\section{Discussion}

COVID-19 is a global pandemic spreading in almost every country of the world. Saudi Arabia announced its first case on March 2, 2020. This study describes the clinical characteristics and outcome of laboratory confirmed COVID-19 patients in a single center. Although it is a small study, it reflects the various clinical aspects of COVID-19 patients. Several risk factors for severe COVID-19 have been reported in recent literature which includes advanced age, Hypertension, immunocompromised state, diabetes mellitus, cardiovascular disease, chronic lung disease, chronic kidney disease, liver disease, malignancy and morbid obesity [12-14]. The trend of more severe disease, in this study, in older patients with co-morbid conditions, is the same as reported in studies from China and other countries. Although the frequency of respiratory symptoms in this group of patients is in line with other published case series [15] the rate of non-pulmonary symptoms was strikingly high including fatigue, anorexia, anosmia, ageusia and headache [16-18]. Disorder of smell has been reported as 3-fold higher in those testing positive for COVID-19 than those testing negative [19]. There are reports of seizure disorder [20], acute hepatitis [21] and encephalopathy [22] related to SARS CoV-2 virus infection. Slightly elevated liver enzymes are not uncommon in symptomatic patients with COVID-19 especially in severe disease and it is presumed to be caused by direct effect of virus infection of liver cells [23]. Common laboratory findings in this study included lymphopenia, high neutrophil to lymphocyte ratio and elevated levels of C-reactive protein, ferritin and D-dimer. The pattern of laboratory findings was the same as reported earlier. The rise in inflammatory markers and degree of lymphopenia was correlated with severity of disease. The rate of co-infection was low as compared to published studies [24]. Only one patient had co-infection with Moraxella Catarrhalis. Common radiological finding was ground glass, focal or scattered and patients with severe disease typically had bilateral opacities. Majority of symptomatic patients received hydroxychloroquine and Azithromycin [25] unless contraindicated due mainly to prolonged QT inter- val. Anti-viral therapy including Lopinavir/Ritonavir (Kaletra) and Ribavirin was used in patient in early period but after the update of institution guidelines, patients admitted in the later period of study, did not receive this regimen. Patient with clinical and radiological evidence of pneumonia received empirical broad spectrum antibiotics. Patients with critical disease received one or more of additional experimental medications such as IL-6 receptor blocker, Tocilizumab [26] and intravenous immunoglobulin (IVIG) [27]. Thirty-five (69\%) patients recovered and were discharged while $16(31 \%)$ were still at the hospital. None of the patient at the time of study conclusion was in ICU. Mean ( $\pm S D$ ) duration of time to negative COVID-19 RT-PCR from symptom onset was 21 ( \pm 7.3 ) days. The time from symptom onset to negative RT-PCR was longer than what is reported in many studies [28].

\section{Conclusion}

This study reflects the early experience with COID-19 in Saudi Arabia and describes clinical, laboratory, radiology features and clinic outcome. Severe disease was more common in older patients and those with co-morbid conditions. The co-infection rate was low and the time to negative RT-PCR from symptom onset was longer than what was reported in other countries. The study also highlights the exceptionally high rate of non-pulmonary symptoms.

\section{Limitation of Study}

The main limitation of the study is the small sample size and results of this study may not reflect the true picture of a large cohort. The pharmacological agents used for the treatment of COVID-19 in this cohort were not proven in randomized controlled trials and were used as per institutional guidelines for management of COVID-19 patients.

\section{Conflict of Interest}

None.

\section{Funding}

No funding was obtained for this study.

\section{Authors List and Author Contribution}

Sarfraz Saleemi: Concept and design of study, draft writing, analysis of data.

Mohammed Alhajji: Concept and design of study, interpretation of data.

Reem Almaghrabi: Draft review and revision for important intellectual content and data interpretation.

Fahad Alrabiah: Draft review and revision for important intellectual content and data interpretation.

Faisal Albaiz: Acquisition of data or analysis and interpretation of data.

Rayan Alfadda: Concept and design of study, data interpretation.

Jameela Edathodu: Concept and design of study, data interpretation. 


\section{Confirmation of Authors Review}

Manuscript have been read by all authors and approved.

\section{References}

1. WHO (2020) Coronavirus disease (COVID-19) pandemic.

2. (2020) Coronavirus (COVID-19) disease pandemic- statistics \& facts. The Statista Portal.

3. Zheng J (2020) SARS-CoV-2: An emerging coronavirus that causes a global threat. Int J Biol Sci 16: 1678-1685.

4. Zhou P, Yang XL, Wang XG, et al. (2020) A pneumonia outbreak associated with a new coronavirus of probable bat origin. Nature 579: 270-273.

5. Huang C, Wang Y, Li X, et al. (2020) Clinical features of patients infected with 2019 novel coronavirus in Wuhan, China. The Lancet 395: 497-506.

6. Wu Z, Mc Googan JM (2020) Characteristics of and important lessons from the coronavirus disease 2019 (COVID-19) outbreak in China: Summary of a report of 72314 cases from the Chinese Center For Disease Control and Prevention. JAMA 323: 12391242.

7. CDC (2020) Coronavirus disease 2019 (COVID-19) - People who are at higher risk for severe illness.

8. Young BE, Ong SWX, Kalimuddin S, et al. (2020) Epidemiologic features and clinical course of patients infected with SARS-CoV-2 in Singapore. JAMA 323: 1488-1494.

9. Wang D, Hu B, Hu C, et al. (2020) Clinical characteristics of 138 hospitalized patients with 2019 novel Coronavirus-infected pneumonia in Wuhan, China. JAMA 323: 1061-1069.

10. Kanne JP (2020) Chest CT findings in 2019 Novel Coronavirus (2019-nCoV) infections from Wuhan, China: Key points for the radiologist. Radiology 295: 16-17.

11. (2020) Infectious Diseases Society of America Guidelines on the treatment and management of patients with COVID-19. Infectious Diseases Society of America (IDSA).

12. Zhou F, Yu T, Du R, et al. (2020) Clinical course and risk factors for mortality of adult in patients with COVID-19 in Wuhan, China: A retrospective cohort study. Lancet 395: 1054-1062.

13. Jordan Rachel E, Adab Peymane, Cheng KK (2020) Covid-19: Risk factors for severe disease and death. BMJ.

14. Wang K, Zhang Z, Yu M, et al. (2020) 15-day mortality and associated risk factors for hospitalized patients with COVID-19 in
Wuhan, China: An ambispective observational cohort study. Intensive Care Med, 1-3.

15. Yang W, Cao Q, Qin L, et al. (2020) Clinical characteristics and imaging manifestations of the 2019 novel coronavirus disease (COVID-19): A multi-center study in Wenzhou city, Zhejiang, China. J Infect 80: 388-393.

16. Cristina Menni, Ana Valdes, Maxim B Freydin, et al. (2020) Loss of smell and taste in combination with other symptoms is a strong predictor of COVID-19 infection. medRxiv.

17. Yeo C, Kaushal S, Yeo D (2020) Enteric involvement of coronaviruses: Is faecal-oral transmission of SARS-CoV-2 possible? Lancet Gastroenterol Hepatol 5: 335-337.

18. Gu J, Han B, Wang J (2020) COVID-19: Gastrointestinal manifestations and potential fecal-oral transmission. Gastroenterology 158: 1518-1519.

19. Xydakis MS, Dehgani-Mobaraki P, Holbrook EH, et al. (2020) Smell and taste dysfunction in patients with COVID-19. Lancet Infect Dis S1473-S3099.

20. Filatov A, Sharma P, Hindi F, et al. (2020) Neurological complications of Coronavirus disease (COVID-19): Encephalopathy. Cureus 12: e7352.

21. Chau TN, Lee KC, Yao H, et al. (2004) SARS-associated viral hepatitis caused by a novel corona virus, report of three cases. Hepatology 39: 302-310.

22. Ye $M$, Ren $Y, L v T$ (2020) Encephalitis as a clinical manifestation of COVID-19. Brain Behav Immun.

23. Qingxian Cai, Deliang Huang, Hong Yu. et al. (2020) COVID-19: Abnormal liver function tests. J Hepatol.

24. Kim D, Quinn J, Pinsky B, et al. (2020) Rates of co-infection between SARS-CoV-2 and other respiratory pathogens. JAMA 323: 2085-2086.

25. Gautret $P$, Lagier JC, Parola P, et al. (2020) Hydroxychloroquine and azithromycin as a treatment of COVID-19: Results of an open-label non-randomized clinical trial. Int J Antimicrob Agents.

26. Fu B, Xu X, Wei H (2020) Why tocilizumab could be an effective treatment for severe COVID-19? J Transl Med 18: 164.

27. Felsenstein S, Herbert JA, McNamara PS, et al. (2020) COVID-19: Immunology and treatment options. Clin Immunol 215: 108448.

28. De Chang, G Mo, X Yuan, et al. (2020) Time kinetics of viral clearance and resolution of symptoms in novel Coronavirus infection. Am J Respir Crit Care Med 201: 1150-1152.

DOI: $10.36959 / 719 / 568$

Copyright: (C) 2020 Saleemi S, et al. This is an open-access article distributed under the terms of the Creative Commons Attribution License, which permits unrestricted use, distribution, and reproduction in any medium, provided the original author and source are credited. 